\title{
Utility, Appropriateness of Request and Report of Computed Tomography Scan for the Diagnosis of Paranasal Sinuses Pathologies in a Sub-Saharan Africa Urban Setting
}

\author{
Yann Chris Mannel Eng1* ${ }^{*}$ Armel Philippe Awana ${ }^{2}$, Ambroise Merci Seme Engoumou', \\ François Djomou ${ }^{13}$, Odile Fernande Zeh ${ }^{1,4}$ \\ ${ }^{1}$ Faculty of Medicine and Biomedical Sciences, The University of Yaounde I, Yaounde, Cameroon \\ ${ }^{2}$ Jamot Hospital, Yaounde, Cameroon \\ ${ }^{3}$ Ear, Nose and Throat Unit, Yaoundé University Teaching Hospital, Yaounde, Cameroun \\ ${ }^{4}$ Yaoundé Gyneco-Obstetrical and Pediatric Hospital, Yaounde, Cameroon \\ Email: *yannchrismanneleng88@gmail.com
}

How to cite this paper: Eng, Y.C.M., Awana A.P., Engoumou, A.M.S., Djomou, F. and Zeh, O.F. (2021) Utility, Appropriateness of Request and Report of Computed Tomography Scan for the Diagnosis of Paranasal Sinuses Pathologies in a Sub-Saharan Africa Urban Setting. Open Journal of Radio$\log y, 11,175-186$.

https://doi.org/10.4236/ojrad.2021.114016

Received: October 30, 2021

Accepted: December 13, 2021

Published: December 16, 2021

Copyright $\odot 2021$ by author(s) and Scientific Research Publishing Inc. This work is licensed under the Creative Commons Attribution International License (CC BY 4.0).

http://creativecommons.org/licenses/by/4.0/

(c) (i) Open Access

\begin{abstract}
Background: Computed tomography (CT) Scan is the imaging of choice for the diagnosis of sino nasal (SN) pathologies in sub-Saharan African setting. However, it is often requested by non experienced practitioners. We aimed to describe the utility and the appropriateness of CT Scan request forms and results in the diagnosis of SN pathologies. Method: We conducted a retrospective cross-sectional study in four health facilities in Yaoundé, Cameroun, from October 2018 to September 2019. All patients to whom SN CT Scan was performed were called for an appointment. After informed consent, data on the appropriateness and conformity of the request form were collected and compared to the guidelines of the French society of radiology. CT Scan results were also collected and the quality of the report was evaluated. Results: A total of 206 SN CT Scan request forms and reports were included, with a mean patient age of $37.7 \pm 16.1$ years. The request form was conformed to guidelines in $8.3 \%$ of cases and was found appropriate in $62.6 \%$ of cases. No CT Scan report had all the items required, and 159 (77.2\%) reports were useful. The most common diagnosis in patients with abnormal results was chronic sinusitis (49.5\%) and SN polyp or polyposis (19.4\%). Conclusion: Most of SN CT Scan requested were appropriate and useful. However, the conformity of request forms and reports to the guidelines is low. Suggesting the need for capacity building for the diagnosis of SN pathologies especially for non specialists like general practitioners and nurses.
\end{abstract}




\section{Keywords}

Appropriateness, Conformity, CT Scan Request Form, Report, Sino Nasal Pathologies

\section{Introduction}

Sino nasal pathologies are a group of diseases affecting aerial cavity within the bones of the face or the base of the skull. They can be grouped into three main categories: 1) post-traumatic pathologies; 2) inflammatory diseases including acute and chronic rhinosinusitis and Sino nasal polyposis pattern; 3) tumoral pathologies mostly benign and malignant primitive tumours [1]. These pathologies are especially important and potentially serious diseases because they extend to the central nervous system either through the venous system or by contiguity. They also represent a great public health concern due to their high socioeconomical impact and alteration of quality of life. Indeed, chronic rhinosinusitis patients in United Kingdom were found to have a high level of healthcare service use, out-of-pocket expenditure and productivity loss while it results in an average of 20.6 workdays missed during one year in Canada [2] [3]. In the USA, the disease affects more than $16 \%$ of the population [4]. A similar prevalence was found in Cameroon between 2011 and 2015, with 11.7\% of people affected with chronic rhinosinusitis [5].

Imaging for the diagnosis of sino nasal pathologies is based on standard X-ray, computed tomography (CT) scan, and magnetic resonance imaging (MRI). The usage of X-ray is limited to exploration of intrasinusal liquid, as it underestimates the extent of mucosal and bone lesions. Furthermore, X-ray permits a poor analysis of sphenoidal and ethmoidal sinuses. On the other hand, MRI gives a detailed analysis of paranasal sinuses and their link to encephala and meninges [6]. However, the high cost and the few number centers where MRI can be done in our setting (including urban setting) reduce the accessibility of this exam. CT Scan, therefore, remains the analysis of choice in sub-Saharan Africa setting for the diagnosis of paranasal sinuses pathologies. Due to the lack of specialists, it can be requested by every practitioner, including general practitioners and nurses which may not be trained for the diagnosis of sino nasal diseases. Radiologists are not always experienced in the report of sino nasal CT Scan results. We aimed to study the practice and utility of CT Scan for the diagnosis of paranasal sinuses pathologies in a sub-Saharan urban setting. The results will help to design better strategies for capacity building in the management of sino nasal pathology in order to reduce their burden in our setting.

\section{Method}

\subsection{Study Design and Population}

This was a retrospective cross-sectional study design conducted in radiology and 
medical imaging unit of two public and two private health facilities in Yaoundé, Cameroon, namely the Yaoundé Central Hospital, the Yaoundé Gynaeco-obstetric and pediatric hospital, the Jordan Medical Service and the Centre Médical la Cathédrale. We consecutively included all the paranasal sinuses CT Scan request forms and reports received and delivered in these units between October 2018 and September 2019, with no age restriction. Exclusion criteria were radiologic report not validated and/or request form unreadable.

\subsection{Data Collection}

Patients with paranasal sinuses CT Scan performed during the study period were identified through the units' registries and their phone numbers (or the number of their relatives) were collected. Participants or their relatives were therefore contacted by phone for an appointment in the unit where the exam was done, the patient or relative was asked to come with the CT Scan request form and report. Selection criteria were checked on arrival and a written consent was obtained from patients above 18 years and from parents of children below 18 years before inclusion.

The conformity of CT Scan request form was assessed according to the French National Authority of Health (NAH) guidelines for the conformity of imaging exams requests, which recommend that an imaging request form most have 08 items necessary for the exam and results interpretation: five administrative criteria (date of the request, name and unit of the practitioner requesting, name and age or date of birth of the patient) and three clinical criteria (anatomical region to be analysed, imaging indication and purpose or research question) [7]. Clinical data were collected from the request form and grouped in symptoms, signs, syndromes, clinical diagnosis, past history, clinical purpose of the exam and other information. These data were compared with the appropriateness criteria define by the French society of radiologist (FSR) in 05 categories: appropriate (exam usually appropriate for the diagnosis and the treatment in the context), appropriate in special cases (described in the commentary of the document), specialized exam (done on the request of an experienced practitioner), not appropriate (exam not justified for the condition or symptoms cited) and contraindicated exams. Appropriate exams were classified into grade of recommendation $(A, B, C$ or $D)$ as described by the FSR [8]. Grade A: Level of evidence 1 study; Grade B: Level 2 and 3 study or extrapolation of level 1 studies; Grade C: Level 4 study or extrapolation from level 2 or 3 studies; Grade D: Level of evidence 5 or non-conclusive studies at all levels. Level of evidence 1: Systematic review of cross-sectional studies conducted blind and using a reference standard applied systematically; Level of evidence 2: Cross-sectional study conducted blind and using a reference standard applied systematically; Level of evidence 3: Non-consecutive recruiting study; study without systematic application of a reference standard; Level of evidence 4: Case-control study; study using a non-independent or low-quality reference standard; Level of evidence 5: Deductive reasoning. 
The results of the CT Scan (radiological diagnosis, location of lesions and anatomical variants) were collected from the report and the quality of reporting was assessed using the 18 criteria described by the FSR. An exam was considered useful when it was done according to the protocol recommended for the condition, the semiology of lesions was presented on the report and there was an answer to the clinical question asked on the request form (if there was such a question on the request form) [9].

\subsection{Statistical Analysis and Sample Size Calculation}

The minimum sample size was estimated at 93 request forms and reports, using a precision of $10 \%$, a type one error of $5 \%$ and an estimated proportion of appropriate imaging of 59.5\% found by Moifo et al. [10]. Data collected were entered in a data entry application designed in Census and surveys processing system (CsPro) version 7.0. Qualitative data were described as number and percentage while continuous data were described as mean \pm standard deviation. The analysis was performed with IBM Corp. Released 2015. IBM SPSS Statistics for Windows, Version 23.0. Armonk, NY: IBM Corp.

\subsection{Ethical Consideration}

Prior to patient inclusion, authorization was obtained from the directors of the corresponding hospitals and an ethical clearance was obtained from the institutional review board of the faculty of medicine and biomedical sciences, University of Yaoundé 1 (Number: 260/UY1/FMSB/VDRC/CSD). Informed written consent was obtained from all the participants aged above 18 years or from parents of participants below 18 years old. We didn't ask for the assent of children because there were not directly involved in data collection. Data were managed anonymously to preserve confidentiality. The study was conducted according to the ethical principles of medical research involving human subjects, stated in the Helsinki declaration and next versions [11].

\section{Results}

\subsection{Description of the Study Population}

A total of 206 CT Scan request forms and reports were included in the study. Females were more frequent (53.9\%) and the mean age of patients was $37.7 \pm$ 16.1 years, ranging from 1 to 86 years (Table 1). Most of the exams were requested by specialists (53.9\%), mostly otorhinolaryngologists (41.7\%), and the practitioner requesting for the analysis was not specified in 27 (13.1\%) cases. Table 1 gives more details on the study population.

\subsection{Conformity of the CT Scan Request form}

Conformity criteria are described in Table 2 . All the 08 items recommended by the NAH guidelines were found on 17 (8.3\%) request forms (Table 2). The patient's name and age, and the anatomical region to be analyzed were present on 
Table 1. Distribution of the study population according to the requesting practitioners and the patients' characteristics.

\begin{tabular}{|c|c|c|}
\hline Variables & Number & Percentage \\
\hline \multicolumn{3}{|l|}{ Requesting practitioners } \\
\hline General practitioner & 47 & 22.8 \\
\hline Resident & 19 & 9.2 \\
\hline Nurses/Imaging technician & 2 & 1 \\
\hline Specialist & 111 & 53.9 \\
\hline Otorhinolaryngologist & 86 & 41.7 \\
\hline Dental surgeon & 3 & 1.5 \\
\hline Neurologist & 2 & 1 \\
\hline Other specialists & 19 & 9.2 \\
\hline Unspecified & 27 & 13.1 \\
\hline \multicolumn{3}{|l|}{ Gender (patients) } \\
\hline Male & 95 & 46.1 \\
\hline Female & 111 & 53.9 \\
\hline \multicolumn{3}{|l|}{ Age ranges (patients), years } \\
\hline$<10$ & 8 & 3.9 \\
\hline$[10-20[$ & 21 & 10.2 \\
\hline$[20-30[$ & 36 & 17.5 \\
\hline$[30-40[$ & 44 & 21.4 \\
\hline$[40-50[$ & 45 & 21.8 \\
\hline$[50-60[$ & 34 & 16.5 \\
\hline$[60-70[$ & 15 & 7.3 \\
\hline$\geq 70$ & 3 & 1.5 \\
\hline
\end{tabular}

Table 2. Description of the quality of the CT Scan request forms and the appropriateness of the request.

\begin{tabular}{lcc}
\hline Variables & Number & Percentage \\
\hline CT Scan request forms content & 206 & 100 \\
Name of the patient & 205 & 99.5 \\
Anatomical region to be analyzed & 203 & 98.5 \\
Age or date of birth of the patient & 147 & 71.4 \\
Unit of the practitioner requesting & 146 & 70.9 \\
Date of the request & 145 & 70.4 \\
Imaging indication & 51 & 24.8 \\
Purpose or research question & 27 & 13.1 \\
Name of the practitioner requesting & & \\
\hline
\end{tabular}


Continued

All the items of the NAH guidelines specified on the form

Yes

17

No

189

Type of clinical information provided on the request forms

Clinical diagnosis

Symptoms

Syndromes

Clinical signs

Clinical purpose

Other information

Past history

No clinical information

Pertinence of the request

Yes

No

Couldn't be assessed ${ }^{*}$

\section{Appropriateness}

Appropriate

Appropriate in special cases

Specialized examination

Not appropriate

Couldn't be assessed ${ }^{*}$

\section{Grade of recommendation of CT Scan}

Grade B

Grade C

Not appropriate/not assessed ${ }^{*}$

76 36.4

The quality of the CT Scan request forms was described according to the French NAH guidelines for the conformity of imaging exams requests. The appropriateness of the request was described according to Guide for proper use of medical imaging examinations of the French Society of Radiology. CT: Computed Tomography; NAH: National Authority for Health. ${ }^{\star}$ The item couldn't be assessed due to absent or insufficient information for the assessment.

almost all the request forms; while the purpose of the exams and the name of the requesting practitioner were reported on less than $25 \%$ of request forms (Table 2). Clinical information provided on forms was mainly clinical diagnosis, symptoms and syndromes, respectively in 35\%, 18.4\% and 17\% of request forms. However, no clinical information was present on $19.9 \%$ of request forms (Table 2). 


\subsection{Appropriateness of the Request}

The CT Scan requested for paranasal sinuses pathologies diagnosis was found pertinent for 129 (62.6\%) patients; the request was not appropriate or the appropriateness couldn't be evaluated for 75 (36.4\%) cases (Table 2). Among those which were appropriate, 110 (53.4\%) were Grade B recommendation (Table 2).

\subsection{Quality of CT Scan Report and Utility}

All the CT Scan reports contained patient identification, radiologist identity, date of report writing, anatomical region examined results of the exam and the validator signature. No report had the date on which the exam was done, and $11.2 \%$ reported the date of report validation (Table 3). Characteristics of radiocontrast

Table 3. Description of the quality of radiological reporting after CT Scan for paranasal sinuses pathologies in the study population.

\begin{tabular}{|c|c|c|}
\hline Variables & Number & Percentage \\
\hline \multicolumn{3}{|l|}{ CT Scan report content } \\
\hline Patient identification & 206 & 100 \\
\hline Radiologist identity & 206 & 100 \\
\hline Date of the report writing & 206 & 100 \\
\hline Anatomical region examined & 206 & 100 \\
\hline Results of the exam & 206 & 100 \\
\hline Validator signature & 206 & 100 \\
\hline Conclusion of the report & 203 & 98.5 \\
\hline Technic used & 186 & 90.3 \\
\hline Requesting practitioner & 174 & 84.5 \\
\hline Indication of the exam & 167 & 81.1 \\
\hline Equipment used & 42 & 2.04 \\
\hline Dosimetry & 37 & 18 \\
\hline Date of report validation & 23 & 11.2 \\
\hline Type of radiocontrast agent used $\left(\mathrm{n}=19^{\star}\right)$ & 1 & 5.3 \\
\hline Volume of radiocontrast agent used $\left(n=19^{\star}\right)$ & 1 & 5.3 \\
\hline Concentration of radiocontrast agent $\left(n=19^{\star}\right)$ & 1 & 5.3 \\
\hline Date of the exam & 0 & 0 \\
\hline \multicolumn{3}{|l|}{ Utility } \\
\hline Semiological description on the report & 206 & 100 \\
\hline Exam done according to the recommended protocol for the question & 161 & 78.2 \\
\hline Conclusion answering the question on the request & 160 & 77.7 \\
\hline Useful exam & 159 & 77.2 \\
\hline
\end{tabular}

The quality of radiologic report was described according to the guidelines of the French Society of Radiology. ${ }^{\star}$ Radiocontrast agent was used for 19 patients. CT: Computed Tomography. 
agent administration was described in 5.3\% (1/19) of patients who need those agents for the exam. As described in Table 3, the CT Scan was found useful for 159 (77.2\%) patients.

\subsection{Radiological Profile of Study Population}

The CT Scan was normal for $52(25.2 \%)$ patients. The most common diagnosis in patients with abnormal results were chronic sinusitis and sino nasal polyp/polyposis (Table 4). These pathologies were mostly found in the maxillary and the anterior ethmoidal sinuses, respectively for 153 (74.3\%) and 104 (50.5\%) cases (Table 4). At least one anatomical variant was observed in 49 (23.8\%) results, Concha bullosa (12.1\%) and septal deviation with bone spur $(8.3 \%)$ been the more common. See Table 4 for more details on radiological profile.

Table 4. Description of the results of CT Scan in the study population.

\begin{tabular}{|c|c|c|}
\hline Variables & Number & Percentage \\
\hline \multicolumn{3}{|l|}{ Radiological diagnosis } \\
\hline Chronic sinusitis & 102 & 49.5 \\
\hline Sino nasal polyp/polyposis & 39 & 19.4 \\
\hline Aspergillosis & 6 & 2.9 \\
\hline Tumor & 5 & 2.4 \\
\hline Acute sinusitis & 3 & 1.5 \\
\hline Other diagnosis & 18 & 8.7 \\
\hline Normal CT Scan result & 52 & 25.2 \\
\hline \multicolumn{3}{|l|}{ Lesions topography } \\
\hline Maxillary sinus & 153 & 74.3 \\
\hline Anterior ethmoidal sinus & 104 & 50.5 \\
\hline Frontal sinus & 90 & 43.7 \\
\hline Sphenoidal sinus & 85 & 41.3 \\
\hline Posterior ethmoidal sinus & 61 & 29.6 \\
\hline No lesion & 52 & 25.2 \\
\hline \multicolumn{3}{|l|}{ Anatomical variants } \\
\hline Concha bullosa & 25 & 12.1 \\
\hline Septal deviation with bone spur & 17 & 8.3 \\
\hline Carotid protrusion & 8 & 3.9 \\
\hline AggerNassi & 4 & 1.9 \\
\hline Clinoid apophyse pneumatisation & 2 & 1 \\
\hline Dehiscence of the planum bone & 1 & 0.5 \\
\hline No anatomical variant & 157 & 76.2 \\
\hline
\end{tabular}

CT: Computed Tomography. 


\section{Discussion}

CT Scan like other imaging methods are intended to provide accurate and complete identification of relevant findings, a coherent opinion of the radiologist concerning the underling cause of abnormalities and if appropriate a guidance on other investigation to be done. However, an appropriate request form and a good report are required for these purposes. In some sub-Saharan Africa settings, CT Scan is a key element for diagnosis of paranasal sinuses pathologies. The request forms are often done by non specialist and the results report by non experienced radiologists. We found in this study that less than $10 \%$ of request forms had all the required items, about two third of the requests were appropriate and the CT Scan was found useful in $77.2 \%$ of cases.

Among the 206 request forms included in this study, the name and age of the patient was written on almost all the forms; a result found also by Roussel et al. [12]. Indeed, the identity of the patient is the first element requested on the form, and define the subject to whom the exam should be done. The name of the requesting practitioner was absent on $86.9 \%$ of reports. Moifo et al. found a better result, with $22.9 \%$ of request were that name was absent [10]. The difference can be explained by the fact that in our study, patients were coming from any health facility in the town while in the other study, practitioners were in the same hospital as the radiology unit, and could be identified and sanctioned if the request form was not good. Nevertheless, the proportion of request forms without the name of practitioner remain high in both cases, and may be due to lack of confidence and/or ability to prescribe the exam. Our results show that only $53.6 \%$ of those practitioners were specialists which have been trained to request the CT Scan of paranasal sinuses.

In our population, $62 \%$ of request were pertinent and $63.6 \%$ were appropriate for the patients. This result is close to what was found by Stanescu et al. and Lehnert et al. In both studies, about $70 \%$ of request for imaging were appropriate [13] [14]. Similarities can be related to the prescribing practitioners which included general practitioners in those studies and in our population. The proportion of inappropriate CT Scan request is still high, suggesting the need of capacity building for those who prescribe this exam, especially in our low resource setting where most of the patients have to pay out pocket. These inappropriate requests constitute a source of wasting resources, and may explain the 52 (25\%) normal CT Scan results found in our population.

No report was contained all the required item for a good imaging result report, yet all these reports were validated by a senior radiologist. The dosimetry and the equipment used for the analysis were among the less reported items, present respectively on $11.2 \%$ and $18 \%$ of the reports. Although the radiation dosimetry is required to inform the level of exposure to ionisation and thus the risk from the CT Scan especially for children which may have a lifetime risk of fatal cancer as high as 1 in 1000 cases. The radiation dosimetry is also intend to assess the degree of optimisation of radiologic procedure in the unit [15]. Radi- 
ologist should be aware that these easy to obtain information are really important both for the patients' protection and the radiology unit improvement. Other key elements which were absent on the reports are the characteristics of radiocontrast agents used for the exam. Indeed, they were reported for only $5 \%$ of the patients. Knowing that adverse events after administration of radiocontrast media can occur up to one week after injection-especially in young adult and women which were more frequent in this population-lacking information on the type, the concentration and the volume of radiocontrast media will really hamper management of these patients in case of adverse events [16].

The overall utility of the CT Scan was good in $77.2 \%$ of cases, which is lower than the $87.5 \%$ of useful CT Scan found by Chin et al. concerning non traumatic acute abdominal pain [17]. This difference may be related to the easiest identification of lesions on abdominal CT Scan compare to paranasal sinuses analysis. Furthermore, the utility of the exam may have been further altered by the poor quality of request forms and finding reports. For an efficient usage of resources, all these components of the CT Scan results chain should be improved in our context.

This study presents some limitations including the fact that requesting practitioner was not contacted for more information in order to better assess the appropriateness of the request, nor the patient's file used for this purpose. This approach would have produced more reliable results concerning the real appropriateness of the requested exams. Another limitation can be the exclusion of non validated results, as they may also represent poor reporting practices. We choose to exclude this population because non validated results may have been produced out of the normal path of results in the facility.

\section{Conclusion}

CT Scan requested for diagnosis of paranasal sinuses pathologies were useful for less than $80 \%$ of patients. The request forms were delivered by non specialists in about half of cases, and were not appropriate for $63.6 \%$ of patients. No report of findings contained all the required items, with some key elements like dosimetry and characteristics of radiocontrast agent used absent in about $90 \%$ of reports. These results suggest the need for capacity building of all the practitioners who may request CT Scan and radiologist in charge of interpretating and validating results in our setting. In order to efficiently use the resources which are sparse, and to improve the security of patients in case of adverse events.

\section{Conflicts of Interest}

The authors declare no conflicts of interest regarding the publication of this paper.

\section{References}

[1] Stelow, E.B. (2015) Sinonasal and Nasopharyngeal Pathology. In: Wick, M.R., Li- 
Volsi, V.A., Pfeifer, J.D., Stelow, E.B. and Wakely, P.E.J., Eds., Silverberg's Principles and Practice of Surgical Pathology and Cytopathology. 5e éd. Cambridge University Press, Cambridge, 1125-1177. https://doi.org/10.1017/9781139137201.016

[2] Wahid, N.W., Smith, R., Clark, A., Salam, M. and Philpott, C.M. (2020) The Socioeconomic Cost of Chronic Rhinosinusitis Study. Rhinology, 58, 112-125. https://doi.org/10.4193/Rhin19.424

[3] Yip, J., Vescan, A.D., Witterick, I.J. and Monteiro, E. (2017) The Personal Financial Burden of Chronic Rhinosinusitis: A Canadian Perspective. American Journal of Rhinology \& Allergy, 31, 216-221. https://doi.org/10.2500/ajra.2017.31.4452

[4] Shashy, R.G., Moore, E.J. and Weaver, A. (2004) Prevalence of the Chronic Sinusitis Diagnosis in Olmsted County, Minnesota. Archives of Otorhinolaryngology-Head \& Neck Surgery, 130, 320-323. https://doi.org/10.1001/archotol.130.3.320

[5] Liale Honore, T. (2016) Prevalence and Management of Rhinosinusitis in Developing Countries: The Case of Mbingo Baptist Hospital, North West Region, Cameroon. Texila International Journal of Clinical Research, 3, 47-66. https://doi.org/10.21522/TIJCR.2014.03.02.Art004

[6] Ferrie, J.-C., Fontanel, J.-P., Delagranda, A., Dufour, X. and Klossek, J.-M. (2008) Imagerie radiologique des cavités sinusiennes et nasales. Radiologie et imagerie médicale, 3, 1-19. https://doi.org/10.1016/S1879-8535(08)72770-3

[7] Indicateur Conformité des demandes d'examens d'imagerie [Internet]. France: Haute Autorité de Santé; 2011 juill [cité 3 mars 2021]p.28.

https://www.has-sante.fr/plugins/ModuleXitiKLEE/types/FileDocument/doXiti.jsp? $\underline{\mathrm{id}=\mathrm{c} \_1098040}$

[8] (2013) Société Française de Radiologie, Société Française de Médecine Nucléaire. Guide du Bon Usage des examens d'imagerie médicale [Internet].

http://gbu.radiologie.fr/

[9] Groupe de travail SFR_CRR. (2007) Recommandations générales pour l'élaboration d'un compte-rendu radiologique (CRR). Journal de Radiologie, 88, 304-306. https://doi.org/10.1016/S0221-0363(07)89822-2

[10] Moifo, B., Ndeh Kamgnie, M., Ninying Fuh, F., Zeh, O., Tebere, H., Moulion Tapouh, J., et al. (2013) Pertinence of Indications of Medical Imaging Examinations at Yaounde-Cameroon. Health Sciences and Disease, 14, 1-8.

[11] (2013) World Medical Association Declaration of Helsinki: Ethical Principles for Medical Research Involving Human Subjects. JAMA, 310, 2191-2194. https://doi.org/10.1001/jama.2013.281053

[12] Roussel, N. and Lelièvre, P. (2002) Améliorer la qualité des prescriptions d'examens d'imagerie médicale. European Journal of Radiology, 83, 621-625.

[13] Stanescu, G., Rosca-Fartat, G. and Stanescu, D. (2015) Justification of CT Scans Using Referral Guidelines for Imaging. Radiation Protection Dosimetry, 165, 43-46. https://doi.org/10.1093/rpd/ncv060

[14] Lehnert, B.E. and Bree, R.L. (2010) Analysis of Appropriateness of Outpatient CT and MRI Referred from Primary Care Clinics at an Academic Medical Center: How Critical Is the Need for Improved Decision Support? Journal of the American College of Radiology, 7, 192-197. https://doi.org/10.1016/j.jacr.2009.11.010

[15] Wall, B.F. (2004) Radiation Protection Dosimetry for Diagnostic Radiology Patients. Radiation Protection Dosimetry, 109, 409-419. https://doi.org/10.1093/rpd/nch317

[16] Bellin, M.-F., Stacul, F., Webb, J.A.W., Thomsen, H.S., Morcos, S.K., Almén, T., et 
al. (2011) Late Adverse Reactions to Intravascular Iodine Based Contrast Media: An Update. European Radiology, 21, 2305-2310.

https://doi.org/10.1007/s00330-011-2200-9

[17] Chin, J.Y., Goldstraw, E., Lunniss, P. and Patel, K. (2012) Evaluation of the Utility of Abdominal CT Scans in the Diagnosis, Management, Outcome and Information Given at Discharge of Patients with Non-Traumatic Acute Abdominal Pain. The British Journal of Radiology, 85, e596-e602. https://doi.org/10.1259/bjr/95400367 